\title{
VSG-Based Parameter Adaptive Control Strategy
}

\author{
Guo Jianyi ${ }^{1, *}$, Fan Youping ${ }^{1}$ \\ ${ }^{1}$ School of Electrical Engineering and Automation, Wuhan University, Wuhan, Hubei, China
}

\begin{abstract}
As a large number of converters composed of power electronic devices are connected to the grid, power system has gradually decreased stability. How to increase dynamic response of the converter has become one of the research hotspots. Virtual synchronous generator technology (VSG) can endow the converter with moment of inertia and damping characteristics, thereby enhancing dynamic response, but the traditional VSG technology cannot achieve the optimal control effect. To solve this problem, an adaptive control strategy is proposed, which takes logical combination of system angular velocity and frequency change as the real-time change condition, with exponential function as the change expression. Finally, this paper uses MATLAB / Simulink to compare the method in this paper with several existing typical control strategies.
\end{abstract}

\section{Introduction}

In recent years, distributed power generation technology has attracted much attention. Under this background, converters composed of power electronic devices have been widely used, which reduces the inertia of the system as a whole and impacts the dynamic response and stable operation of the system. Hence, it is a hot research topic to improve the converter's dynamic response, so that it possesses the running characteristics of synchronous generator. In this way, virtual synchronous generator technology emerges as the time requires ${ }^{[1]}$.

At present, researchers have done a lot of research on VSG technology. In traditional control methods, adaptive control strategies are designed based on ping-pong algorithm and fuzzy control algorithm ${ }^{[2-3]}$, but the parameters controlled by the ping-pong algorithm cannot be changed continuously, so there is still room for improvement. Although the fuzzy control algorithm perfects the ping-pong algorithm to a certain extent, it is essentially a refinement of the former. In addition, some scholars have solved the deficiencies of fuzzy control algorithms by controlling parameter change according to a certain function law ${ }^{[4-5]}$, but the control effect is not ideal due to the failure to make full use of the moment of inertia and damping.

Aiming at the shortcomings of the existing VSG technology, this paper proposes a control strategy based on exponential function, which makes full use of the influence of the moment of inertia and damping on the system. For its basic principle: Based on the conventional VSG technology, the reasonable change trend of damping and moment of inertia is analyzed under system disturbance, and an adaptive control strategy in gridconnected mode is designed in combination with exponential function. Through simulation comparison with three existing control strategies, it indicates that the strategy of this paper can better maintain system stability.

\section{Introduction}

\subsection{The basic principle of virtual synchronous generator}

\subsubsection{Virtual Synchronous Machine Model}

The electrical equation and the second-order motion equation of synchronous generator are as follows ${ }^{[6]}$ :

$$
\left\{\begin{array}{c}
e=U+i(R+j X) \\
T_{m}-T_{e}=\frac{P_{m}}{\omega}-\frac{P_{e}}{\omega}=J \frac{d \omega}{d t}+D \Delta \omega \\
\Delta \omega=\omega-\omega_{0} \\
d \theta / d t=\omega
\end{array}\right.
$$

Where, $R$ represents stator resistance, $X$ represents reactance, $U$ represents armature terminal voltage, $T_{m}$ represents mechanical torque, $T_{e}$ represents electromagnetic torque, $D$ represents damping characteristics, $J$ represents moment of inertia, $\omega$ represents mechanical angular velocity, $\omega_{0}$ represents rated angular frequency, $\Delta \omega$ represents the deviation between actual and rated angular velocity, and $\theta$ is the output power angle.

In the VSG ontology model, the angular velocity is calculated by $P e, P m$. The differential of the electrical angle is angular velocity. The product of the electrical angle and $E_{0}$ is taken as the subsequent input. Substitute (1) and the terminal voltage of the virtual synchronous generator is obtained. Then, the calculation result is

\footnotetext{
*Corresponding author: Jianyi Guo@3110495468@qq.com
} 
submitted into the voltage and current loop and modulated to generate the corresponding trigger pulse to be delivered to the converter, as shown in Figure 1.

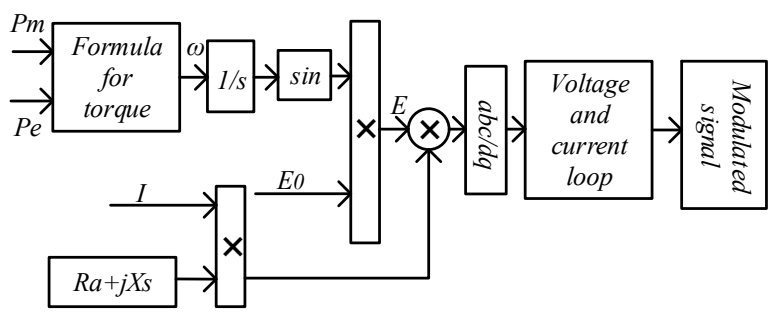

Fig. 1. Model structure diagram of VSG ontology

\subsubsection{Power frequency and excitation control}

Drawing lessons from the principles of synchronous generator governor and excitation regulator, we design the active power regulation and reactive voltage characteristic equations, which are expressed as

$$
\left\{\begin{array}{l}
P_{e}=P_{r e f}+K_{P}\left(\omega_{r e f}-\omega\right) \\
U_{r e f}=E_{0}+D_{q}\left(Q_{r e f}-Q\right)
\end{array}\right.
$$

In the formula, $K_{p}$ is the active frequency droop coefficient, $P_{\text {ref }}$ is the given active power, $\omega$ is the output angular frequency value, $\omega_{\text {ref }}$ is the rated angular frequency, $P_{e}$ is the virtual mechanical power, $U_{r e f}$ is the amplitude of the given voltage, $E_{0}$ is the rated voltage amplitude, $Q_{\text {ref }}$ is the rated value of reactive power, $D_{q}$ is the reactive power adjustment coefficient, and $Q$ is the output reactive power.

\subsubsection{The overall system structure}

The virtual synchronous generator system is composed of DC source $\mathrm{V}_{\mathrm{dc}}$, voltage-type converter, filter circuit, load and $\mathrm{AC}$ bus. First, collect the signals including main circuit output voltage, current, active power, reactive power to generate modulation signals through the virtual speed governor, excitation controller, voltage current dualloop controller, and finally generate the pulse signal to control the converter, as shown in Figure 2.

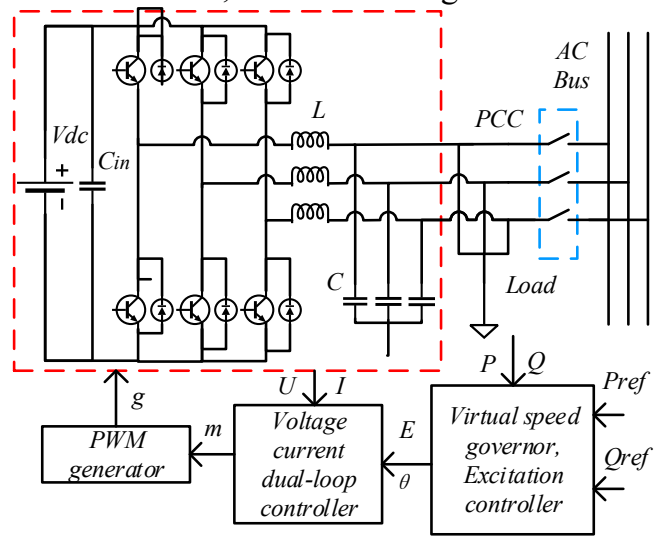

Fig. 2. The overall control structure of the VSG system

\subsection{Principle analysis of the adaptive model}

By increasing the moment of inertia, it is possible to reduce the frequency change rate and suppress the maximum frequency deviation, but adjustment time will be longer. However, by increasing the damping, it is also possible to reduce the maximum frequency deviation and shorten the time to peak, so better results can be achieved via combination of the two.

When the system is disturbed, the angular velocity $\Delta \omega$ changes over time as is shown in Figure 3 . It is divided into five time points corresponding to $t_{0}, t_{1}, t_{2}, t_{3}$ and $t_{4}$. Next, we will analyze the system requirements for moment of inertia and damping in each section.

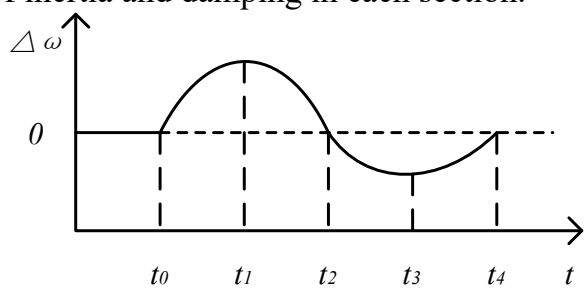

Fig3 Chart of changes in $\Delta \omega$ over time

Section $t_{0} \sim t_{1}$ : The given system power increases at $t_{0}$, with the angular frequency of the system increased and electrical energy converted into kinetic energy. Meanwhile, $d \omega / d t$ increases abruptly from 0 . In this section, $d \omega / d t>0$ and $\Delta \omega>0$. The output angular frequency of the system reaches the maximum at $t_{1}$. At this time, the moment of inertia should be increased to restrain the further increase in system overshoot, while damping should be reduced to make the angular velocity of the system reach the peak as soon as possible.

Section $t_{1} \sim t_{2}$ : At this time, the system $d \omega / d t<0$, so the angular frequency returns from the maximum value to the initial system value between $t_{1} \sim t_{2}, \Delta \omega>0$. A smaller moment of inertia and bigger damping are needed to rapidly achieve steady state.

The analysis process of sections $t_{2} \sim t_{3}$ and $t_{3} \sim t_{4}$ are similar to that of the previous two sections.

According to the above analysis, the results are shown in Table 1.

Table 1. Results of the analysis

\begin{tabular}{|c|c|c|c|c|c|}
\hline Interval & $\Delta \boldsymbol{\omega}$ & $/ \boldsymbol{d} \boldsymbol{\omega} / \boldsymbol{d} \boldsymbol{t} /$ & $\begin{array}{c}\Delta \boldsymbol{\omega}^{*} \\
/ \boldsymbol{d} \boldsymbol{\omega} / \boldsymbol{d} \boldsymbol{t} /\end{array}$ & $\boldsymbol{J}$ & $\boldsymbol{D}$ \\
\hline$t_{0} \sim t_{1}$ & $>0$ & $>0$ & $>0$ & Big & Small \\
\hline$t_{1} \sim t_{2}$ & $>0$ & $<0$ & $<0$ & Small & Big \\
\hline$t_{2} \sim t_{3}$ & $<0$ & $<0$ & $>0$ & Big & Small \\
\hline$t_{3} \sim t_{4}$ & $<0$ & $>0$ & $<0$ & Small & Big \\
\hline
\end{tabular}

\subsection{Establishment of adaptive model}

In this paper, exponential function model is used to establish the function model of adaptive moment of inertia, as shown in (3).

$$
J= \begin{cases}J_{0} & \left|\frac{d f}{d t}\right|<N_{J} \cup \Delta \omega \frac{d f}{d t}<0 \\ J_{0}+k_{1}\left(\left|\frac{d f}{d t}\right|\right)^{m 1} & \left|\frac{d f}{d t}\right|>N_{J} \cap \Delta \omega \frac{d f}{d t}>0\end{cases}
$$

Where, $J_{0}$ is the initial value of the moment of inertia, $N_{J}$ is the set value of the frequency change rate, the 
proportional coefficient and exponential coefficient are $k_{1}$ and $m_{1}$, respectively.

Through small signal analysis of the system, we obtain mathematical relationship between damping and moment of inertia, and the function model of damping can be further obtained:

$$
D=\left\{\begin{array}{cc}
D_{0} & \Delta \omega \frac{d f}{d t}>0 \cup\left|\frac{d f}{d t}\right|<N_{D} \\
D_{0} \sqrt{1+\frac{1}{J_{0}} k_{1}\left(\left|\frac{d f}{d t}\right|\right)^{m 1}} & \Delta \omega \frac{d f}{d t}<0 \cap\left|\frac{d f}{d t}\right|>N_{D}
\end{array}\right.
$$

The thresholds $N_{J}, N_{D}$ are set mainly to avoid that the data in the adaptive model still changes frequently when the system is stable, causing judgment disorder and failure.

\subsection{Three existing strategies}

Strategy 1: The parameters are constant, that is, the moment of inertia and damping coefficient of the system are constant values.

Strategy 2: Moment of inertia is adaptive, that is, make improvement based on strategy 1 , so that moment of inertia of the system implements adaptive strategy, while the damping coefficient is maintained at a fixed value.

Strategy 3: The collaborative strategy proposed in one of the references ${ }^{[7]}$, which is a result of improvement based on strategy 2 . The moment of inertia and damping coefficient of the system are both adaptively changed. The law of change is shown in Table 2.

Table 2. Analysis results of Strategy 3

\begin{tabular}{|c|c|c|c|c|}
\hline Interval & $t_{0} \sim t_{1}$ & $t_{1} \sim t_{2}$ & $t_{2} \sim t_{3}$ & $t_{3} \sim t_{4}$ \\
\hline $\boldsymbol{J}$ & Big & Small & Big & Small \\
\hline $\boldsymbol{D}$ & Big & Small & Big & Small \\
\hline
\end{tabular}

To reflect the advantages of the adaptive strategy in this paper, the initial values of the moment of inertia and damping coefficient of the four strategies are kept consistent. The adaptive change functions required for strategy 2 and strategy 3 are the same as those proposed in this paper.

\section{Results \& Discussion}

To verify the adaptive control strategy proposed above, a simulation model is built in Matlab/Simulink, and the relevant control parameters of this paper are shown in Table 3.

Table 3. Parameter settings

\begin{tabular}{|c|c|c|c|}
\hline Parameter & Value & Parameter & Value \\
\hline $\begin{array}{c}\text { Rated } \\
\text { capacity }\end{array}$ & $8000 \mathrm{Var}$ & $\begin{array}{c}\text { Operating } \\
\text { frequency }\end{array}$ & $10 \mathrm{kHz}$ \\
\hline DC voltage & $700 \mathrm{~V}$ & $k_{\mathrm{p}}$ & 10000 \\
\hline $\begin{array}{c}\text { Filter } \\
\text { inductance }\end{array}$ & $0.01 \mathrm{H}$ & $k_{\mathrm{q}}$ & 0.002 \\
\hline $\begin{array}{c}\text { Filter } \\
\text { capacitor }\end{array}$ & $7.04 \times 10^{-6} \mathrm{~F}$ & $U_{\text {gabc }}$ & $311 \mathrm{~V}$ \\
\hline Line & $0.01 \Omega$ & Grid side & $0.1 \Omega$ \\
\hline
\end{tabular}

\begin{tabular}{|c|c|c|c|}
\hline resistance & & resistance & \\
\hline $\begin{array}{l}\text { Grid's side } \\
\text { inductance }\end{array}$ & $0.002 \mathrm{H}$ & $f_{0}$ & $50 \mathrm{~Hz}$ \\
\hline$P_{\text {set }}$ & $8000 \mathrm{~W}$ & $Q_{\text {set }}$ & 0 \\
\hline$m_{1}$ & 1.5 & $k_{1}$ & 2.5 \\
\hline$J_{0}$ & 0.25 & $D_{0}$ & 2 \\
\hline$N_{\mathrm{J}}$ & 0.5 & $N_{\mathrm{D}}$ & 0.5 \\
\hline
\end{tabular}

The system is integrated into the grid before $0.2 \mathrm{~s}$, and the given power $P_{\text {set }}$ decreases from $8 \mathrm{~kW}$ to $5 \mathrm{~kW}$ at 0.2 $\mathrm{s}$, and then increases to $15 \mathrm{~kW}$ at $0.7 \mathrm{~s}$. The comparison results between frequency and active power of the three strategies mentioned above and the strategy in this paper are shown in Figure 4 and Figure 5 respectively.

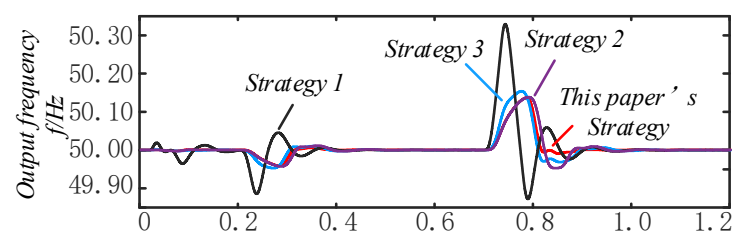

Fig. 4. Comparison result of output frequency

As shown in Figure 4, compared with the other three strategies, the strategy proposed in this paper has comparable output frequency overshoot as strategy 3 , both of which are superior to the other two strategies. Compared with strategy 3, this paper's strategy has the advantage of fast entry into stable state, thus reducing the shock time.

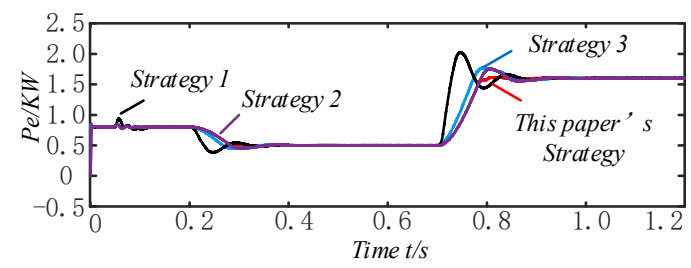

Fig. 5. Comparison result of output active power

As shown in Figure 5, when $P_{\text {set }}$ changes, compared with the other three strategies, the strategy in this paper almost has no overshoot in output active power, and enters the steady state earlier. This is a control effect unachievable for the other three strategies.

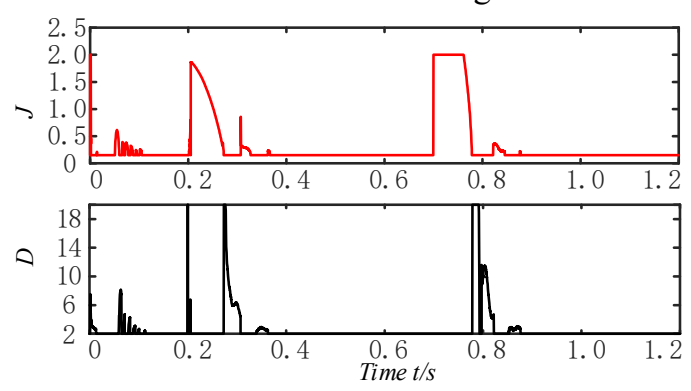

Fig. 6. Wave of J and D

Figure 6 is the image reflecting changes in the moment of inertia $J$ and damping $D$ of the system under the control strategy of this paper. It can be seen that the moment of inertia and damping are staggered and adaptive, which conforms to the design theory of this paper. The topping 
phenomenon during the change process is due to the amplitude limiting link added in the strategy. Owing to the sudden change of $|d f / d t|$, spikes appear during the change of virtual moment of inertia $J$ and damping coefficient $D$.

In summary, compared with the other three control strategies, the strategy of interleaved control of moment of inertia and damping proposed in this paper can reduce the overshoot of the system output frequency, power and current, reduce the system oscillation time, and improve system stability.

\section{Conclusions}

To improve the dynamic response performance of VSG, this paper designs an adaptive control strategy based on the exponential function, which comprehensively considers the influence of moment of inertia and damping on the system to give full play to the advantages of the two. Also, a simulation model is established, with simulation results indicating that:

The magnitude of moment of inertia and damping can affect the output characteristics of the entire system, and by selecting appropriate parameters, it is possible to improve response characteristics of the system. Compared with the existing three control strategies, the adaptive strategy proposed in this paper significantly reduces system vibration and optimizes the transient response process.

At the same time, this paper also has the following shortcomings: it temporarily stays at the simulation level without physical verification; DC source is used in replace of the energy storage system, so further improvements are needed to adapt to the actual situation.

\section{References}

1. Shi, R L., Zhang, X., Xu, H Z., et al. (2016) Seamless Switching Control Strategy for Microgrid Operation Modes Based on Virtual Synchronous Generator. J. Automation of Electric Power Systems., 40 (10): 1623

2. Zhu, Z B., Huang, S P., Li, Z X., et al. (2020) Research on Control Strategy of Micro-grid Adaptive Rotating Inertia Virtual Synchronous Generator. J. Proceedings of the CSU-EPSA., 32(04): 111-115.

3. Yang, Y., Mei, F., Zhang, C Y., et al. (2019) Coordinated adaptive control strategy of rotational inertia and damping coefficient for virtual synchronous generator. J. Electric Power Automation Equipment., 39(03): 125-131.

4. Ying, G L., Dong, H., Dai, Y C., et al. (2020) Adaptive Control Strategy of VSG Parameters in Photovoltaic Microgrid. J. Power System Technology., 44(01): 192-199.

5. Li, Y Z., Guo, X., Dong, H Y., et al. (2020) VSG Control Strategy for Adaptive Moment of Inertia in Island Micro-grid. J. Proceedings of the CSU-EPSA., 32(12): 144-150.

6. Lv, Z P., Sheng, W X., Zhong, Q C., et al. (2014)
Virtual Synchronous Generator and Its Applications in Micro-grid. J. Proceedings of the CSEE, 34(16): 2591-2603.

7. Zhu, Z B., Huang, S P. (2020) Microgrid Stability Control Based on Adaptive Rotating Inertia VSG. J. Journal of Electrical Engineering, 15(01): 41-47. 